\title{
Pneumococcal vaccination and risk of myocardial infarction
}

\author{
François Lamontagne MD MSc, Marie-Pierre Garant PhD, Jean-Christophe Carvalho MD, \\ Luc Lanthier MD MSc, Marek Smieja MD PhD, Danielle Pilon MD MSc
}

$\infty \quad$ See related commentary by Madjid, page 749

\section{ABSTRACT}

Background: Based on promising results from laboratory studies, we hypothesized that pneumococcal vaccination would protect patients from myocardial infarction.

Methods: We conducted a hospital-based case-control study that included patients considered to be at risk of myocardial infarction. We used health databases to obtain hospital diagnoses and vaccination status. We compared patients who had been admitted for treatment of myocardial infarction with patients admitted to a surgical department in the same hospital for a reason other than myocardial infarction between 1997 and 2003.

Results: We found a total of 43209 patients who were at risk; of these, we matched 999 cases and 3996 controls according to age, sex and year of hospital admission. Cases were less likely than controls to have been vaccinated (adjusted odds ratio [OR] $0.53,95 \%$ confidence interval $[\mathrm{Cl}]$ $0.40-0.70$ ). This putative protective role of the vaccine was not observed for patients who had received the vaccine up to 1 year before myocardial infarction (adjusted OR 0.85 , $95 \% \mathrm{Cl}$ 0.54-1.33). In contrast, if vaccination had occurred 2 years or more before the hospital admission, the association was stronger (adjusted OR 0.33, 95\% Cl 0.20-0.46).

Interpretation: Pneumococcal vaccination was associated with a decrease of more than $50 \%$ in the rate myocardial infarction 2 years after exposure. If confirmed, this association should generate interest in exploring the putative mechanisms and may offer another reason to promote pneumococcal vaccination.

Une version française de ce résumé est disponible à l'adresse www.cmaj.ca/cgi/content/full/179/8/773/DC1

CMAJ 2008;179(8):773-7

$\mathrm{D}$ espite important advances in primary prevention, atherosclerosis remains the leading cause of death in developed societies. ${ }^{1}$ In addition to risk factors such as hypertension, diabetes mellitus, tobacco use and dyslipidemia, less traditional risk factors have also been sought. Many markers, including C-reactive protein and interleukins, highlight inflammation as a key mediator in both the progression and activation of atherosclerotic lesions. ${ }^{2-4}$ Some medications that are used to prevent cardiovascular diseases, such as statins, also appear to reduce inflammation. ${ }^{5}$
Animal experiments have shown that pneumococcal vaccination reduces the extent of atherosclerotic lesions. ${ }^{6} \mathrm{We}$ hypothesized that antibodies directed against Streptococcus pneumoniae also recognize oxidized low-density lipoprotein (LDL) and impede the formation of foam cells. Interestingly, a retrospective cohort study involving World War II veterans who had undergone splenectomy documented excess mortality rates from both pneumonia and ischemic heart disease. More recent data have suggested that acute pneumococcal infections, but not vaccinations, increase the risk of vascular events; ${ }^{8}$ however, the duration of vaccination exposure considered in that study was limited.

Our primary objective was to evaluate the association between pneumococcal vaccination and the risk of myocardial infarction. We also explored whether any effect of vaccination on the risk of infarction waned over time.

\section{Methods}

\section{Design and ethics approval}

We conducted a case-control study of patients who were considered at risk for myocardial infarction and who had been admitted to a tertiary care hospital. We obtained approval for this study from the research ethics board of the Centre hospitalier universitaire de Sherbrooke and Quebec's Commission d'accès à l'information.

\section{Data sources}

We used 2 databases for this study. The first was the research-purpose database ${ }^{9}$ of the Centre informatisé de recherche évaluative en services et soins de santé of the Centre hospitalier universitaire de Sherbrooke, a tertiary care teaching hospital in the province of Quebec. Along with demographic data, this database included, for each hospital admission since 1996, detailed information on all primary and secondary diagnoses, coded according to the International Classification of Diseases, 9th revision (ICD-9). This database also contained all biochemical and pharmaceutical data recorded during the admission, including, for each medication prescribed, the name, dosage, formulation, quantity dis-

From the Department of Medicine (Lamontagne, Garant, Carvalho, Lanthier, Pilon), Université de Sherbrooke, Sherbrooke, Que.; and the Department of Clinical Epidemiology and Biostatistics (Lamontagne, Smieja), McMaster University, Hamilton, Ont. 
pensed, date dispensed and duration of prescription. Other researchers have used this database in previous pharmacoepidemiologic studies. ${ }^{10-12}$

We also used the Logivac database, a governmentmaintained database that records each pneumococcal vaccination administered in the province of Quebec. Since 1988, the Agence de la santé et des services sociaux de l'Estrie, the public health agency of the Estrie area, has recorded all such data for the region in this database.

\section{Study population}

We considered for inclusion all patients who were at risk for myocardial infarction and who were admitted to the Centre hospitalier universitaire de Sherbrooke between Jan. 1, 1997, and Dec. 31, 2003. We defined risk for myocardial infarction as the presence of at least one of the following risk factors, as recorded in the patients' medical records: high blood pressure, diabetes mellitus or dyslipidemia in men older than 45 years and in women older than 50 years. We included only patients who had a permanent address in the same administrative area as the Centre hospitalier universi-

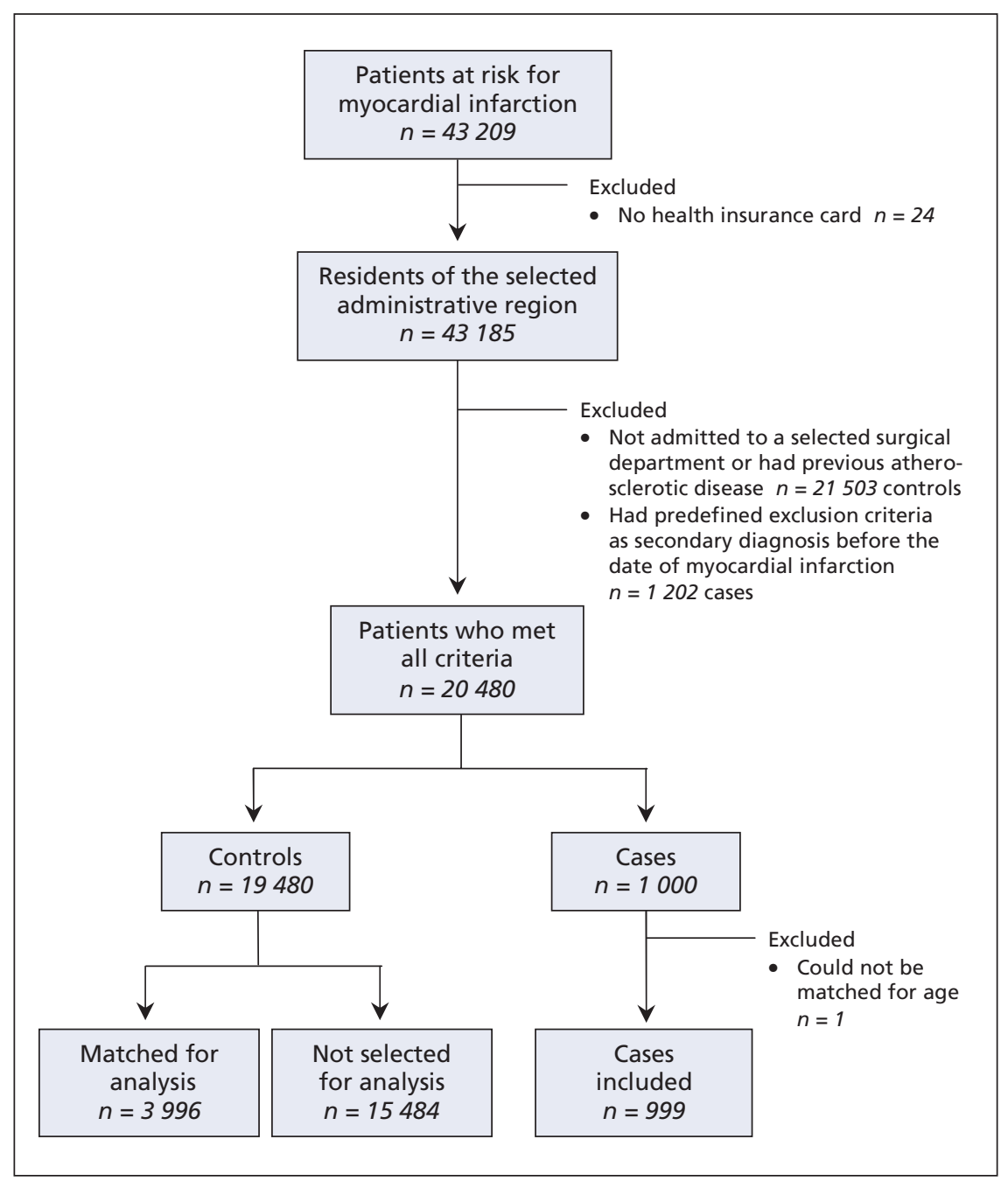

Figure 1: Flow diagram showing the selection of cases and controls for inclusion in a study of the relation between pneumococcal vaccination and myocardial infarction. taire de Sherbrooke, where this hospital is the sole dispenser of specialized care.

We defined cases as patients with one or more of the specified cardiovascular risk factors, as documented in the medical record, and no previously diagnosed atherosclerotic disease (i.e., coronary, cerebral or peripheral vascular disease, vascular dementia or any revascularisation) who had experienced a new (i.e., incident) myocardial infarction within the study period. We identified myocardial infarction with or without ST-segment elevation on the basis of ICD-9 code 410. The hospital's research database did not provide data on deaths from myocardial infarction; thus, we did not differentiate between episodes that resulted in death and those that did not. For cases, we deemed the date of hospital admission for myocardial infarction to be the index date.

We defined controls as patients with one or more of the specified cardiovascular risk factors, as documented in the medical record, and no previously diagnosed atherosclerotic disease (i.e., coronary, cerebral or peripheral vascular disease, vascular dementia or any revascularisation), who did not experience a new myocardial infarction during the study period.

We included only patients who had been admitted to one of the hospital's surgical departments (orthopedics, gynecology, urology, general surgery, plastic surgery or otorhinolaryngology). For controls, we considered the date of admission into one of these departments as the index date. We matched 4 controls to each case on the basis of age (within 2 years), sex and index date (within 1 year).

\section{Definition of exposure}

We considered patients to have been exposed to the pneumococcal polysaccharide vaccine if they had received this vaccine in the 10 years before the index date, as recorded in the Logivac database. We stratified exposure by the interval between vaccination and index date: up to and including 1 year before the index date, more than 1 year but less than 2 years before the index date, and 2 years or more before the index date. We were unable to perform further stratification because too few patients had received the vaccine between 3 and 10 years before the index date. These cut-offs were arbitrary, as there are no clinical data to support this classification.

\section{Definition of other important risk factors}

We included the following variables in our models (as recorded in the patients' medical records between 1997, when the computerized database was implemented, and the index date): chronic obstructive pulmonary disease, chronic renal failure, his- 
tory of splenectomy, history of S. pneumoniae infection, diabetes mellitus, hypertension and dyslipidemia. We selected these covariables because we considered them a priori as likely confounders; that is, associated with both myocardial infarction and pneumococcal vaccination. We based our choices on biological rationales, rather than statistically significant associations.

\section{Statistical analysis}

On the basis of the findings from a pilot study, we estimated baseline exposure to the pneumococcal polysaccharide vaccine at $20 \%$. Given a type 1 error of 0.05 , a type 2 error of 0.2 , a correlation coefficient for exposure of 0.9 and an odds ratio (OR) of 0.5, representing a moderate to large effect size, we needed a sample size of 4610 patients: 922 cases and 3688 controls. An OR of less than 1 implies that the odds of being exposed to the vaccine were lower for cases than for controls, suggesting a protective effect of the vaccine.

We used conditional logistic regression to estimate adjusted OR and 95\% confidence interval (CI) for new myocardial infarctions in relation to receipt of the pneumococcal polysaccharide vaccine. We conducted both univariable and multivariable analyses, incorporating the covariables stated above. We first ran a multivariable model entering in a single step the following covariates: pneumococcal vaccination status, chronic obstructive pulmonary disease, chronic renal failure, previous $S$. pneumoniae infection, splenectomy and diabetes. In our second model, we included in a single step the same covariables along with hypertension and dyslipidemia. For all analyses, we matched cases and controls by age, sex and index date.

\section{Results}

Using information from the Centre informatisé de recherche évaluative en services et soins de santé database, we initially identified 43209 patients at risk for myocardial infarction during the study period. Of these, we selected 999 cases and 3996 controls, matched for age, sex and index date, for analysis (Figure 1).

Overall, cases were significantly less likely than controls to have been vaccinated $(7.1 \%$ v. $11.6 \%)$, and cases were significantly more likely to have chronic renal failure $(10.1 \% \mathrm{v}$. $3.0 \%$ ) and diabetes (15.4\% v. 5.0\%) (Table 1).

The mean time between the administration of the pneumococcal polysaccharide vaccine and the index date was 1.81 years (standard deviation [SD] 1.02) for patients who had been exposed to the vaccine. The mean interval since exposure to the vaccine was 1.50 (SD 0.95) years before the index date for cases and 1.86 (SD 1.03) years before the index date for controls $(p<0.001)$.

After adjustment for potential confounding variables, cases were significantly less likely than controls to have received pneumococcal vaccine (OR 0.53, 95\% CI 0.40-0.70). With stratification by time since vaccination, this difference was evident for vaccinations given more than 1 year before the index date (Table 2).

Two additional variables (hypertension and dyslipidemia), for which data are not presented in Table 1 or Table 2, were independently associated with the outcome both before and after adjustment. However, including them in the multivariable analysis did not alter the direction or the size of the vaccine effect (OR 0.43, 95\% CI 0.31-0.59).

Table 1: Sociodemographic and health characteristics of cases $(n=999)$ and controls $(n=3996)$ in a study of the relation between pneumococcal vaccine and myocardial infarction

\begin{tabular}{|c|c|c|c|}
\hline \multirow[b]{2}{*}{ Characteristic } & \multicolumn{2}{|c|}{ No. $(\%)$ of patients* } & \multirow[b]{2}{*}{$\begin{array}{c}\text { Odds ratio } \\
(95 \% \mathrm{Cl}) \dagger\end{array}$} \\
\hline & $\begin{array}{c}\text { Cases } \\
n=999\end{array}$ & $\begin{array}{l}\text { Controls } \\
n=3996\end{array}$ & \\
\hline Age, yr, mean (SD) & $59.2(12.7)$ & $58.8(13.0)$ & NA \\
\hline Sex, male & $684(68.5)$ & $2736(68.5)$ & NA \\
\hline Pneumococcal vaccination & $71 \quad(7.1)$ & $465(11.6)$ & $0.55(0.42-0.72)$ \\
\hline \multicolumn{4}{|l|}{ Timing of vaccination in relation to index date, yr } \\
\hline$\leq 1$ & $25 \quad(2.5)$ & $112(2.8)$ & $0.89(0.57-1.38)$ \\
\hline $1-2$ & $46 \quad(4.6)$ & $353(8.8)$ & $0.47(0.34-0.65)$ \\
\hline$\geq 2$ & $20 \quad(2.0)$ & $208(5.2)$ & $0.36(0.22-0.57)$ \\
\hline \multicolumn{4}{|l|}{ Concurrent conditions } \\
\hline Chronic obstructive pulmonary disease & $41 \quad(4.1)$ & $66(1.7)$ & $1.70(0.77-3.75)$ \\
\hline Chronic renal failure & $101(10.1)$ & $119(3.0)$ & $3.97(2.97-5.31)$ \\
\hline History of Streptococcus pneumoniae infection & $7 \quad(0.7)$ & $21(0.5)$ & $1.33(0.57-3.14)$ \\
\hline Splenectomy & $8 \quad(0.8)$ & $7 \quad(0.2)$ & $4.57(1.66-12.61)$ \\
\hline Diabetes mellitus & $154(15.4)$ & $200(5.0)$ & $3.59(2.85-4.52)$ \\
\hline
\end{tabular}

Note: $\mathrm{Cl}=$ confidence interval, $\mathrm{NA}=$ not applicable, $\mathrm{SD}=$ standard deviation

*Unless indicated otherwise.

tOdds ratios for myocardial infarction were estimated from univariable conditional logistic regression models (for matched analyses) between risk of myocardial infarction and each of the independent variables (total $n=4995$ ). 
Table 2: Adjusted odds ratios for myocardial infarction associated with pneumococcal vaccination among patients at risk for cardiovascular diseases $(n=4995)$

Comparison; adjusted odds ratio* $(95 \% \mathrm{Cl})$

\begin{tabular}{|c|c|c|c|c|}
\hline \multirow[b]{2}{*}{ Risk factor } & \multicolumn{4}{|c|}{ 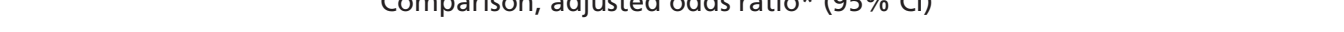 } \\
\hline & $\begin{array}{c}\text { Vaccine } \\
\text { (v. no vaccine) }\end{array}$ & $\begin{array}{c}\text { Vaccine } \leq 1 \text { yr before index } \\
\text { date ( } v \text {. no vaccine } \\
\text { or vaccine }>1 \text { yr before } \\
\text { index date) }\end{array}$ & $\begin{array}{c}\text { Vaccine }>1 \mathrm{yr} \text { before index } \\
\text { date ( } \mathrm{v} \text {. no vaccine } \\
\text { or vaccine } \leq 1 \mathrm{yr} \text { before } \\
\text { index date) }\end{array}$ & $\begin{array}{c}\text { Vaccine } \geq 2 \text { yr before index } \\
\text { date }(v . \text { no vaccine } \\
\text { or vaccine }<2 \text { yr before } \\
\text { index date) }\end{array}$ \\
\hline Pneumococcal vaccination & $0.53(0.40-0.70)$ & $0.85(0.54-1.33)$ & $0.46(0.32-0.64)$ & $0.33(0.20-0.46)$ \\
\hline $\begin{array}{l}\text { Chronic obstructive } \\
\text { pulmonary disease }\end{array}$ & $2.21(1.44-3.39)$ & $2.23(1.46-3.42)$ & $2.21(1.44-3.39)$ & $2.24(1.45-3.44)$ \\
\hline Chronic renal failure & $3.17(2.33-4.31)$ & $3.19(2.35-4.33)$ & $3.13(2.30-4.25)$ & 3.08 (2.27-4.18) \\
\hline $\begin{array}{l}\text { History of Streptococcus } \\
\text { pneumoniae infection }\end{array}$ & $0.67(0.26-1.73)$ & $0.65(0.25-1.69)$ & $0.67(0.26-1.73)$ & $0.68(0.27-1.75)$ \\
\hline Splenectomy & $2.61(0.84-8.17)$ & $2.85(0.91-8.88)$ & $2.62(0.84-8.21)$ & $2.62(0.83-8.23)$ \\
\hline Diabetes mellitus & $3.36(2.65-4.27)$ & $3.26(2.57-4.13)$ & $3.38(2.66-4.29)$ & $3.43(2.70-4.36)$ \\
\hline
\end{tabular}

Note: $\mathrm{Cl}=$ confidence interval.

*In each of the 4 models, each odds ratio was adjusted for the other risk factors in the model.

\section{Interpretation}

Inferences from our study lend support to the hypothesis that vaccination against $S$. pneumoniae is associated with a lower risk of myocardial infarction. Our results suggest that, after a number of confounding and modifying variables were taken into account, the odds of having received a vaccination against $S$. pneumoniae in the group who had experienced myocardial infarction was about half that in the control group. Moreover, this association appeared stronger and the benefit appeared to increase with time since exposure to the vaccine.

Several studies have examined the effect of infections on atherosclerosis. Specific antibiotic regimens targeting a single microbe and given as secondary prevention did not reduce the incidence of acute coronary syndromes. ${ }^{13-16}$ Some authors have suggested that it is the total burden of infection at various sites and the associated inflammatory cascade that affect the progression of atherosclerosis and elicit clinical manifestations., ${ }^{4,1718}$ A recent study ${ }^{8}$ showed a greater risk of myocardial infarction and stroke after acute respiratory and urinary tract infection and a lower risk after influenza vaccination. In that study, in contrast to our study, pneumococcal vaccination was not associated with a lower risk of myocardial infarction; however, the vaccination history was limited to 91 days before the vascular episode.

In addition to preventing acute $S$. pneumoniae infections, the pneumococcal vaccine is thought to alter the natural history of atherosclerosis in another way. In pathogen-free mice without the LDL receptor, vaccination against $S$. pneumoniae decreased the extent of atherosclerotic lesions in the aorta by 30 weeks. ${ }^{6}$ This phenomenon appeared to result from molecular mimicry between the recognized epitopes on S. pneumoniae and oxidized LDL. IgM antibodies directed against $S$. pneumoniae also impeded the uptake of oxidized LDL by macrophages, thereby interrupting an early and crucial step leading to atherosclerosis. The fact that this protective effect of the vaccine appeared after 1 year also concurs with results obtained by Smeeth and colleagues, ${ }^{8}$ who found that pneumococcal vaccination did not alter the risk of a vascular event when it had been given within 3 months before the diagnosis.

Our study had some limitations. First, there may have been residual confounding. Other cardiovascular risk factors, such as smoking, medication use, obesity and lifestyle factors, including exercise and diet, could not be incorporated in the model because these characteristics are not recorded in the research database. It is plausible that patients who are more concerned about their health are less likely to smoke and are more likely to seek vaccination. Another limitation of our study was the potential for misclassification of exposure; specifically, some patients might have received the pneumococcal vaccine outside of the administrative region and their exposure would not have been recorded in the government database. We attempted to minimize this potential problem by selecting only patients who resided in the administrative area. Furthermore, it is unlikely that cases and controls would differ in terms of this factor. Finally, the study population was limited to one centre and consisted mostly of white males, which limits the external validity of our results. Despite these limitations, the strength and precision of the association, as well as the apparent dose-response by time of exposure favour the conclusion that true causality exists. Validation through a larger prospective study is required to help resolve these issues.

In conclusion, the results of this study of patients at risk for vascular disease suggest an effect of pneumococcal vaccination in reducing episodes of new myocardial infarction. Future projects should aim to confirm the association and better characterize the immune and inflammatory responses to the vaccine. 
This article has been peer reviewed.

Competing interests: None declared.

Contributors: François Lamontagne was responsible for initiating the project and contributed to the conception and design of the study and the acquisition and interpretation of the data; he wrote the initial and subsequent versions of the article. Marie-Pierre Garant contributed to the data analysis and interpretation. Jean-Christophe Carvalho contributed to the acquisition and interpretation of the data. Luc Lanthier and Marek Smieja contributed to the design of the study and edited the manuscript. Danielle Pilon supervised the design and interpretation of the data. All of the authors made substantial revisions to the intellectual content of the manuscript and approved the final version.

Acknowledgements: Dr. Sophie Michaud contributed to the design of the study. Hassan Diab was responsible for the management of the Centre informatisé de recherche evaluative en soins et systèmes de la santé database.

Danielle Pilon has received funding from the Fonds de la recherche en santé du Quebec.

Funding: The Quebec Ministère de la santé et des services sociaux and the Agence de la santé et des services sociaux de l'Estrie provided funding for this study.

\section{REFERENCES}

1. Martin GM. Atherosclerosis is the leading cause of death in the developed societies. Am J Pathol 1998;153:1319-20.

2. Ridker PM, Stampfer MJ, Rifai N. Novel risk factors for systemic atherosclerosis: a comparison of C-reactive protein, fibrinogen, homocysteine, lipoprotein(a), and standard cholesterol screening as predictors of peripheral arterial disease. JAMA 2001;285:2481-5

3. Kullo IJ, Gau GT, Tajik AJ. Novel risk factors for atherosclerosis. Mayo Clin Proc 2000;75:369-80.

4. Hansson GK. Inflammation, atherosclerosis, and coronary artery disease. $N$ Engl J Med 2005;352:1685-95.
5. Schönbeck U, Libby P. Inflammation, immunity, and HMG-CoA reductase inhibitors: statins as antiinflammatory agents? Circulation 2004;109(Suppl 1):II18-26.

6. Binder CJ, Horkko S, Dewan A, et al. Pneumococcal vaccination decreases atherosclerotic lesion formation: molecular mimicry between Streptococcus pneumoniae and oxidized LDL. Nat Med 2003;9:736-43.

7. Robinette CD, Fraumeni JF Jr. Splenectomy and subsequent mortality in veterans of the 1939-45 war. Lancet 1977;2:127-9.

8. Smeeth L, Thomas SL, Hall AJ, et al. Risk of myocardial infarction and stroke af ter acute infection or vaccination. N Engl J Med 2004;351:2611-8.

9. Grant A, Moshyk A, Diab H, et al. Integrating feedback from a clinical data warehouse into practice organisation. Int J Med Inform 2006;75:232-9.

10. Brophy JM, Brassard P, Bourgault $C$. The benefit of cholesterol-lowering medications after coronary revascularization: a population study. Am Heart J 2005;150:282-6.

11. Lachaine J, Rinfret S, Merikle EP, et al. Persistence and adherence to cholesterol lowering agents: evidence from Régie de l'assurance maladie du Québec data. Am Heart J 2006;152:164-9.

12. Rahme E, Toubouti $Y$, Hunsche E. Therapy switching and associated costs in elderly patients receiving COX-2 selective inhibitors or non-selective non-steroidal antiinflammatory drugs in Quebec, Canada. Rheumatology (Oxford) 2006;45:903-10.

13. Cannon CP, Braunwald E, McCabe $\mathrm{CH}$, et al. Antibiotic treatment of Chlamydia pneumoniae after acute coronary syndrome. N Engl J Med 2005;352:1646-54.

14. Cercek B, Shah PK, Noc M, et al. Effect of short-term treatment with azithromycin on recurrent ischaemic events in patients with acute coronary syndrome in the Azithromycin in Acute Coronary Syndrome (AZACS) trial: a randomised controlled trial. Lancet 2003;361:809-13.

15. Grayston JT, Kronmal RA, Jackson LA, et al. Azithromycin for the secondary prevention of coronary events. $N$ Engl J Med 2005;352:1637-45.

16. O'Connor CM, Dunne MW, Pfeffer MA, et al. Azithromycin for the secondary prevention of coronary heart disease events: the WIZARD study: a randomized controlled trial. JAMA 2003;290:1459-66.

17. Zhu J, Nieto FJ, Horne BD, et al. Prospective study of pathogen burden and risk of myocardial infarction or death. Circulation 2001;103:45-51.

18. Zhu J, Quyyumi AA, Norman JE, et al. Effects of total pathogen burden on coronary artery disease risk and C-reactive protein levels. Am J Cardiol 2000;85:140-6.

Correspondence to: Dr. Danielle Pilon, Faculty of Medicine, Université de Sherbrooke, 3001, 12e Avenue N, Sherbrooke QC J1H 5N4; fax819 820-6406; Danielle.Pilon@USherbrooke.ca

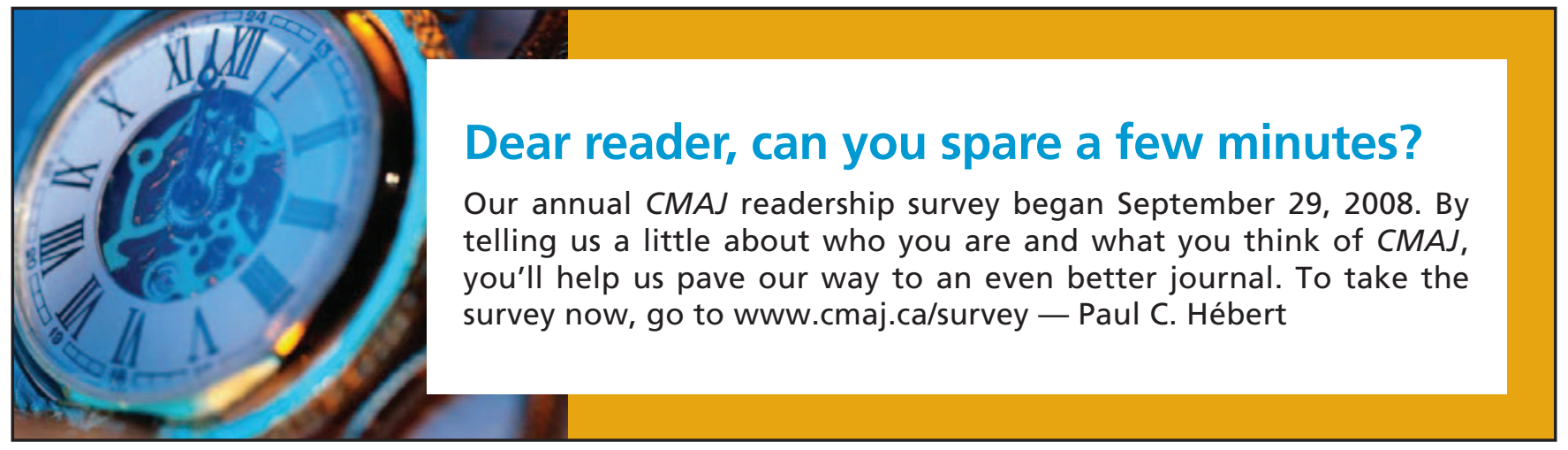

